\title{
Supplemental Information:Surface
}

\section{Transformations of Lead Oxides and Carbonates using First-Principles and Thermodynamics}

\section{Calculations}

Ryan T. Grimes, Joshua A. Leginze, Robert Zochowski, and Joseph W.

$$
\text { Bennett** }
$$

Department of Chemistry and Biochemistry

University of Maryland Baltimore County, Baltimore, MD 21250;

E-mail: bennettj@umbc.edu 


\section{S1: Exchange Correlation Functionals}

Prior work on $\mathrm{PbO}^{1}$ was performed before the inclusion of newly designed and rigorously tested pseudopotentials into the planewave-based open source DFT codes ABINIT $^{2,3}$ and Quantum Espresso, ${ }^{4}$ so we investigated which sets of methodology would work well for $\mathrm{Pb}$ containing oxides and carbonates. In Table S1 we show how the lattice parameters change as a function of differences in methodology by exploring the range of exchange correlation functionals available in 2 open source DFT codes, Quantum Espresso and ABINIT, each of which uses a different set of pseudopotentials. For our Quantum Espresso ${ }^{4}$ analysis we use the $\mathrm{GBRV}^{5}$ set of ultrasoft pseudopotentials ${ }^{6}$ and for $\mathrm{ABINIT}^{2,3}$ we use the $\mathrm{ONCV}^{7}$ set of pseudopotentials.

The following exchange correlation functionals were explored in this work: PBE-GGA, ${ }^{8}$ the van der Waals (vdW) correction of Grimme, ${ }^{9}$ revPBE, ${ }^{10}$ the Wu-Cohen (WC) modified GGA, ${ }^{11}$ and the RPBE of Ref. ${ }^{12}$ Across the board, for our different material/code/potential combinations, the Wu-Cohen GGA performed the best of the set, with all errors between -1 to $+1.7 \%$. revPBE tended to have the largest overestimations of lattice parameters for the set, as did RPBE. The Wu-Cohen GGA also obtained closer lattice parameters to experiment than vdW relaxations of litharge.

\section{S2: Surface Calculation Details}

The smallest litharge supercell slab was of dimensions $1 \times 1 \times 5$, where we have $52 \mathrm{D} \mathrm{Pb}-\mathrm{O}-\mathrm{Pb}$ repeat units, as shown in Figure 1a of the main text. We consider the 3 interior units as bulk-like and the exterior 2 (on either side) as the surface units. As displayed in Table S2, the $1 \times 1 \times 5 \mathrm{~Pb}$-terminated supercell slab had a length $22.561 \AA$ and each slab was separated by $17.616 \AA$ of vacuum to prevent the surfaces from interacting. The largest litharge supercell slab was of dimensions $2 \times 2 \times 7$, where we have $72 \mathrm{D} \mathrm{Pb}$-O-Pb repeat units, as shown in Figure 1a of the main text. We consider the 5 interior units as bulk-like and the exterior 2 
Table S1: Tabulated here are the lattice constants of our exchange correlation tests using the open source codes Quantum Espresso and ABINIT. All entries are reported in units of Angstroms $(\AA)$, and the percent deviation is reported in parentheses. For the ABINIT entries we provide the ixc variable for each exchange correlation functional, where applicable.

\begin{tabular}{|c|c|c|c|}
\hline Espresso & $a(\AA)$ & $b(\AA)$ & $c(\AA)$ \\
\hline \multicolumn{4}{|l|}{ Litharge } \\
\hline Experiment & 3.972 & 3.972 & 5.023 \\
\hline GGA & $4.053(+2.04 \%)$ & $4.053(+2.04 \%)$ & $5.358(+6.67 \%)$ \\
\hline WC-GGA & $3.989(+0.42 \%)$ & $3.989(+0.42 \%)$ & $5.028(+0.10 \%)$ \\
\hline GGA/vdW & $4.041(+1.75 \%)$ & $4.041(+1.75 \%)$ & $5.119(+1.916 \%)$ \\
\hline \multicolumn{4}{|l|}{ Massicot } \\
\hline Experiment & 5.893 & 5.490 & 4.753 \\
\hline WC-GGA & $5.900(+0.08 \%)$ & $5.512(+0.35 \%)$ & $4.719(-0.73 \%)$ \\
\hline \multicolumn{4}{|l|}{ Cerussite } \\
\hline Experiment & 6.134 & 5.180 & 8.492 \\
\hline GGA & $6.298(+2.67 \%)$ & $5.231(+0.99 \%)$ & $8.556(+0.75 \%)$ \\
\hline WC-GGA & $6.073(-1.00 \%)$ & $5.183(+0.06 \%)$ & $8.494(+0.02 \%)$ \\
\hline ABINIT & $a(\AA)$ & $b(\AA)$ & $c(\AA)$ \\
\hline \multicolumn{4}{|l|}{ Litharge } \\
\hline & $(\AA)$ & $(\AA)$ & $(\AA)$ \\
\hline Experiment & 3.972 & 3.972 & 5.023 \\
\hline GGA (11) & $4.084(+2.83 \%)$ & $4.084(+2.83 \%)$ & $5.364(+6.80 \%)$ \\
\hline GGA/vdW (11) & $4.047(+1.89 \%)$ & $4.047(+1.89 \%)$ & $5.262(+4.76 \%)$ \\
\hline revPBE (14) & $4.113(+3.56 \%)$ & $4.113(+3.56 \%)$ & $6.226(+23.97 \%)$ \\
\hline WC-GGA (23) & $4.003(+0.80 \%)$ & $4.003(+0.80 \%)$ & $5.107(+1.69 \%)$ \\
\hline RPBE (15) & $3.878(-2.37 \%)$ & $3.878(-2.37 \%)$ & $8.823(+75.65 \%)$ \\
\hline \multicolumn{4}{|l|}{ Massicot } \\
\hline Experiment & 5.893 & 5.490 & 4.753 \\
\hline GGA (11) & $6.308(+7.00 \%)$ & $5.826(+6.05 \%)$ & $4.812(+1.21 \%)$ \\
\hline revPBE (14) & $8.430(+43.00 \%)$ & $6.055(+10.23 \%)$ & $4.868(+2.40 \%)$ \\
\hline WC-GGA (23) & $5.910(+0.26 \%)$ & $5.504(+0.20 \%)$ & $4.746(+0.16 \%)$ \\
\hline RPBE (15) & $7.871(+33.51)$ & $6.132(+11.64)$ & $4.877(+2.58)$ \\
\hline \multicolumn{4}{|l|}{ Cerussite } \\
\hline Experiment & 6.134 & 5.180 & 8.492 \\
\hline GGA (11) & $6.316(+2.98 \%)$ & $5.216(+0.70 \%)$ & $8.587(+1.12 \%)$ \\
\hline revPBE (14) & $6.469(+4.12 \%)$ & $5.284(+7.27 \%)$ & $8.603(+7.90 \%)$ \\
\hline WC-GGA (23) & $6.073(-1.00 \%)$ & $5.183(+0.06 \%)$ & $8.494(+0.03 \%)$ \\
\hline RPBE (15) & $6.530(+6.45 \%)$ & $5.257(+2.07 \%)$ & $8.636(+1.69 \%)$ \\
\hline
\end{tabular}


(on either side) as the surface units. As displayed in Table S2, the $2 \times 2 \times 7 \mathrm{~Pb}$-terminated supercell slab had a length $32.677 \AA$ and each slab was separated by $17.545 \AA$ of vacuum to prevent the surfaces from interacting.

The smallest massicot supercell slab was of dimensions $3 \times 1 \times 1$, where we have $32 \mathrm{D} \mathrm{Pb}$ $\mathrm{O}-\mathrm{O}-\mathrm{Pb}$ repeat units, as shown in Figure $1 \mathrm{~b}$ of the main text. We consider the 1 interior unit as bulk-like and the exterior 2 (on either side) as the surface units. As displayed in Table S2, the $3 \times 1 \times 1$ Pb-terminated supercell slab had a length $14.500 \AA$ and each slab was separated by $20.075 \AA$ of vacuum to prevent the surfaces from interacting. The largest litharge supercell slab was of dimensions $5 \times 2 \times 1$, where we have $52 \mathrm{D} \mathrm{Pb}-\mathrm{O}-\mathrm{O}-\mathrm{Pb}$ repeat units, as shown in Figure 1b of the main text. We consider the 3 interior units as bulk-like and the exterior 2 (on either side) as the surface units. As displayed in Table S2, the $5 \times 2 \times 1$ Pb-terminated supercell slab had a length $26.289 \AA$ and each slab was separated by $17.076 \AA$ of vacuum to prevent the surfaces from interacting.

Table S2: For each surface calculation discussed in the main text we give the length of the Pb-terminated surface slab $\left(l_{\text {slab }}\right)$ and length of vacuum between cells $\left(l_{\text {vac }}\right)$, both reported in units of $\AA$. Next is the total number of atoms for the Pb-terminated surface, the number of $\mathrm{Pb}$ at the surface $\left(n_{\mathrm{Pb}}\right)$, and finally the \% surface vacancy density that results from exchanging $1 \mathrm{~Pb}$ atom with $2 \mathrm{H}$.

\begin{tabular}{lrrrrr}
\hline & $l_{\text {slab }}$ & $l_{\text {vac }}$ & atoms & $n_{\mathrm{Pb}}$ & $\% \mathrm{~Pb}_{\text {surf }}$ \\
litharge & $(\AA)$ & $(\AA)$ & & & \\
$1 \times 1 \times 5$ & 22.561 & 17.616 & 20 & 1 & $100 \%$ \\
$2 \times 1 \times 5$ & 22.561 & 17.616 & 40 & 2 & $50 \%$ \\
$2 \times 2 \times 5$ & 22.561 & 17.616 & 80 & 4 & $25 \%$ \\
$1 \times 1 \times 7$ & 32.677 & 17.545 & 28 & 1 & $100 \%$ \\
$2 \times 1 \times 7$ & 32.677 & 17.545 & 56 & 2 & $50 \%$ \\
$2 \times 2 \times 7$ & 32.677 & 17.545 & 112 & 4 & $25 \%$ \\
massicot & & & & & \\
$3 \times 1 \times 1$ & 14.500 & 20.075 & 34 & 2 & $50 \%$ \\
$5 \times 1 \times 1$ & 26.289 & 17.076 & 40 & 2 & $50 \%$ \\
$5 \times 2 \times 1$ & 26.289 & 17.076 & 80 & 4 & $25 \%$ \\
cerussite & & & & & \\
$1 \times 1 \times 4$ & 8.398 & 15.906 & 30 & 2 & $50 \%$ \\
$1 \times 1 \times 6$ & 14.368 & 22.088 & 50 & 2 & $50 \%$ \\
$2 \times 1 \times 6$ & 14.368 & 22.088 & 100 & 4 & $25 \%$ \\
$2 \times 2 \times 4$ & 8.398 & 15.906 & 120 & 4 & $25 \%$ \\
\hline
\end{tabular}


The smallest $\mathrm{PbCO}_{3}$ cerussite massicot supercell slab was of dimensions $1 \times 1 \times 4$, where we have 4 total $\mathrm{Pb}$ layers, as shown in Figure 3 of the main text. We consider the 2 interior Pb-layers as bulk-like and the exterior 2 (on either side) as the surface layers. As displayed in Table $\mathrm{S} 2$, the $1 \times 1 \times 4 \mathrm{~Pb}$-terminated supercell slab had a length $8.398 \AA$ and each slab was separated by $15.906 \AA$ of vacuum to prevent the surfaces from interacting. The second largest $\mathrm{PbCO}_{3}$ cerussite massicot supercell slab was of dimensions $2 \times 1 \times 6$, where we have 6 total $\mathrm{Pb}$ layers. We consider the 4 interior Pb-layers as bulk-like and the exterior 2 (on either side) as the surface layers. As displayed in Table S2, the Pb-terminated $2 \times 1 \times 6$ supercell slab had a length $14.368 \AA$ and each slab was separated by $22.088 \AA$ of vacuum to prevent the surfaces from interacting.

\section{S3: DFT + Solvent Ion Thermodynamics}

Here we investigate two routes to release $\mathrm{Pb}$ from the surfaces of litharge, massicot, and cerussite, and use the following systems of equations to determine the thermodynamics of release for a range of $\mathrm{pH}$ values and supercell slab dimensions. Reaction scheme 1 is the exchange of $\mathrm{Pb}$ and $\mathrm{H}$ on the surface of $\mathrm{Pb}$-terminated litharge and massicot surfaces $(\mathrm{PbO} \mathrm{Pb}$-term $)$ to generate $\mathrm{H}$-terminated surfaces $\left(\mathrm{PbO}_{\mathrm{H} \text {-term }}\right)$. The $\mathrm{ZPE}$ added to this reaction depends upon the number $x$ of $\mathrm{H}$ added to create surface $\mathrm{OH}$. Reaction scheme 2 is the removal of surface $\mathrm{Pb}(\mathrm{OH})_{n}$ units from the $\mathrm{PbO}_{\mathrm{H}-\text { term }}$ surface.

$\mathrm{Pb} / \mathbf{H}$ Surface Exchange: $\mathrm{PbO}_{\mathrm{Pb}-\text { term }}+x \mathrm{H} \rightarrow \mathrm{PbO}_{\mathrm{H}-\text { term }}+y \mathrm{~Pb}(\mathbf{1})$

$\Delta G_{1}=E\left(\mathrm{PbO}_{\mathrm{H}-\text { term }}\right)-E\left(\mathrm{PbO} \mathrm{Pb}_{\text {-term }}\right)+y E(\mathrm{~Pb})-x E(\mathrm{H})+\mathrm{ZPE}$

$\Delta G_{2}=y \Delta G_{2}(\mathrm{~Pb})-x \Delta G_{2}(\mathrm{H})$

$\mathrm{Pb}(\mathrm{OH})_{n}$ Removal: $\mathrm{PbO}_{\mathrm{H}-\text { term }} \rightarrow \mathrm{PbO}_{\text {vac }}+\mathrm{Pb}(\mathrm{OH})_{n}(2)$

$\Delta G_{1}=E\left(\mathrm{PbO}_{\text {vac }}\right)-E\left(\mathrm{PbO}_{\mathrm{H}-\text { term }}\right)+E(\mathrm{~Pb})+2 E(\mathrm{O})+2 E(\mathrm{H})+\mathrm{ZPE}$ 
$\Delta G_{2}=E(\mathrm{~Pb})+2 E(\mathrm{O})+2 E(\mathrm{H})$

Using the DFT + Solvent Ion model, where the $\Delta G$ values are given relative to $\mathrm{O}_{2}$ gas, we need to correct for the GGA overestimation of O-O bond dissociation energy. The total energies and differences in energies employed in the current study are presented in Table S3. Table S3: Calculations used to obtain the corrected total energy of an O atom for the DFT + Solvent Ion thermodynamic model. All energies are reported in units of eV.

\begin{tabular}{ll} 
Calculation & $E(\mathrm{eV})$ \\
\hline $\mathrm{O}_{2}, \mathrm{sp} /$ box & -873.364 \\
$\mathrm{O}$ atom, sp/box & -433.355 \\
$\Delta E$ & 6.653 \\
Exp. & 1.406 \\
Corr $\mathrm{O}_{2}$ & -871.958 \\
Corr O & -435.979 \\
\hline
\end{tabular}

In this correction we obtain the difference in total energy between spin polarized $\mathrm{O}_{2}$ and $\mathrm{O}$ atom as $\Delta E=2 E_{\mathrm{O}}-E_{\mathrm{O}_{2}}$. Here our value is $6.653 \mathrm{eV}$, which is $1.406 \mathrm{eV}$ larger than the experimentally determined value of $5.247 \mathrm{eV}$ presented in Ref. ${ }^{8}$ This difference is added to the energy of spin-polarized $\mathrm{O}_{2},\left(\right.$ Corr $\left.\mathrm{O}_{2}\right)$ and then divide by half. The row labeled "Corr O" gives the corrected total energy of an $\mathrm{O}$ atom.

\section{S4: Projected Density of States of Massicot}

The PDOS of massicot surfaces, shown in Figure S1 are similar to the PDOS of litharge surfaces in the main text.

Both surface structures have high O $2 \mathrm{p}$ orbital character in the occupied orbitals and significant $\mathrm{Pb} 6 \mathrm{p}$ character in the unoccupied orbital. Once the surface was protonated, an additional peak was visible around $-7 \mathrm{eV}$ which comes from the new surface $\mathrm{O}$ interaction with $\mathrm{H}$ to form an $\mathrm{OH}$ bond. The H-terminated massicot surface also has more localized electrons than lead terminated, which is seen in both litharge and massicot as a slight increase in band gap, on the order of $\approx 0.1-0.2 \mathrm{eV}$. 


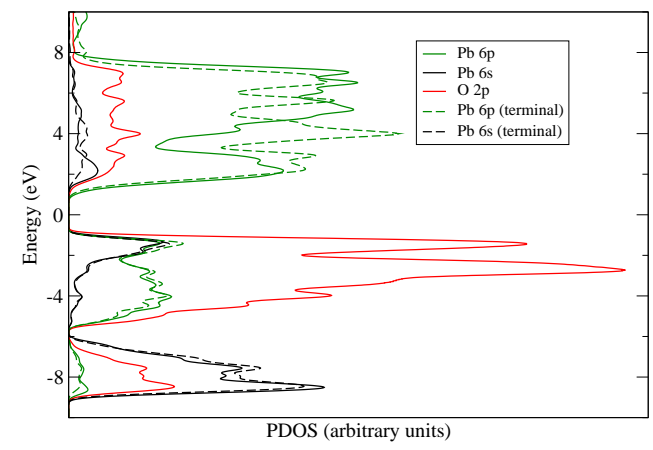

(a) Pb-terminated

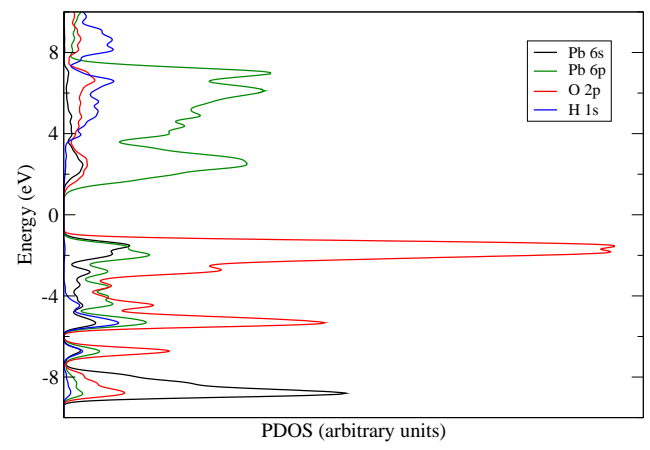

(b) H-terminated

Figure S1: Compared here are the PDOS of $4 \times 1 \times 1$ litharge supercell surfaces that are terminated with a) $\mathrm{Pb}$ and b) $\mathrm{H}$, where each surface $\mathrm{Pb}$ was exchanged for $2 \mathrm{H}$.

\section{References}

(1) Canepa, P.; Ugliengo, P.; Alfredsson, M. Elastic and Vibrational Properties of $\alpha$ - and ß-PbO. J. Phys. Chem. C. 2012, 116, 21514-21522.

(2) Gonze, X.; Amadon, B.; Anglade, P.-M.; Beuken, J.-M.; Bottin, F.; Boulanger, P.; Bruneval, F.; Caliste, D.; Caracas, R.; Cote, M.; et. al., ABINIT: First-principles approach to material and nanosystem properties. Computer Physics Communications 2009, 180, 2582-2615.

(3) Gonze, X.; Jollet, F.; Abreu-Araujo, F.; Adams, D.; Amadon, B.; Applencourt, T.; Audouze, C.; Beuken, J.-M.; Bieder, J.; Bokhanchuk, A.; Bousquet, E.; et al., Recent developments in the ABINIT software package. Computer Physics Communications 2016, 205, 106-131.

(4) Giannozzi, P.; Baroni, S.; Bonini, N.; Calandra, M.; Car, R.; Cavazzoni, C.; Ceresoli, D.; Chiarotti, G. L.; Cococcioni, M.; Dabo, I.; et al., Quantum ESPRESSO: A Modular and Open-Source Software Project for Quantum Simulations of Materials. J. Phys.:Condens. Matter 2009, 21, 395502-20. 
(5) Garrity, K. F.; Bennett, J. W.; Rabe, K. M.; Vanderbilt, D. Pseudopotentials for highthroughput DFT calculations. Comp. Mater. Sci. 2014, 81, 446-452.

(6) Vanderbilt, D. Soft Self-Consistent Pseudopotentials in a Generalized Eigenvalue Formalism. Phys. Rev. B Rapid Comm. 1990, 41, 7892-5.

(7) Hamann, D. R. Optimized norm-conserving Vanderbilt pseudopotentials. Phys. Rev. B. 2013, 88, 085117.

(8) Perdew, J. P.; Burke, K.; Ernzerhof, M. Generalized Gradient Approximation Made Simple. Phys. Rev. Lett. 1996, 77, 3865-8.

(9) Grimme, S. Semiempirical GGA-Type Density Functional Constructed with a LongRange Dispersion Correction. J. Comp. Chem. 2006, 27, 1787-1799.

(10) Zhang, Y.; Yang, W. Comment on "Generalized Gradient Approximation Made Simple”. Phys. Rev. Lett. 1998, 80, 890.

(11) Wu, Z.; Cohen, R. E. More Accurate Generalized Gradient Approximation for Solids. Phys. Rev. B. 2006, 73, 235116.

(12) Hammer, B.; Hansen, L. B.; Nørskov, J. K. Improved adsorption energetics within density-functional theory using Perdew-Burke-Erxerhof functionals. Phys. Rev. B 1999, 59, 7413-21. 